\title{
Restricted Structure Non-linear Generalized Minimum Variance Control of a 2-link Robot Arm
}

\author{
C. Cebeci, M.J. Grimble, R.Katebi and L.F. Recalde
}

\begin{abstract}
The objective of this paper is to propose a restricted structure non-linear generalized minimum variance (RS-NGMV) controller for a two-link robot arm. The NGMV control is a useful method for offering control solutions for nonlinear systems. The motivation is to provide the advantages of NGMV control inside a low-order controller structure with an intention to enable design simplicity and easy implementation for engineers with classical training. The result will be an optimal controller with simple tuning variables. Simulations of the RS-NGMV controller are presented using Matlab/Simulink.
\end{abstract}

\section{INTRODUCTION}

It is the fifth decade of minimum variance (MV) controllers since Åström first introduced them [1]. The strategy was basically to minimize the variance of the stochastic system output. Successful industrial applications of MV controllers came out. However, the controller design was based on the assumption that the plant is of minimum-phase. Results were unstable for non-minimum phase systems. Later, Clarke and Hastings-James [2] proposed the generalized minimum variance (GMV) control extending the MV control law by introducing control costing terms to the cost function. GMV controllers have proven useful in the industry and their self-tuning versions are also available [3]. In 2004, a novel GMV control algorithm was proposed by Grimble [4]. The algorithm was derived for non-linear, multivariable, possibly time varying systems and was called non-linear generalized minimum variance (NGMV) control. It was succeeded by the next generation of NGMV controllers [ $5-12]$ and has shown potential for practical applications [13].

Restricted structure (RS) controllers are characterized by their predefined order and structure. The order and structure of the controllers are independent of the plant order. Typically, these controllers are of lower order than the plant and appear in the form of phase lead, phase lag, phase lead-lag or industrial PID controllers [16]. They are often viewed as low-order approximations to high-order controllers. The fact is, most optimal and predictive controllers are of high order which may make them difficult to implement considering the practical reasons. There comes the need to restrict such advanced control strategies to simpler structures when the design simplicity and the range of applications are taken into account. As a consequence, researchers have taken initiatives $[14,15]$ to use optimal and predictive control methods that have restricted structures.

RS-NGMV control algorithm has been derived recently by Grimble in [17] that is submitted for publication. The

The authors are with the Department of Electronics and Electrical Engineering, University of Strathclyde, Glasgow, UK cagatay.cebeciestrath.ac.uk algorithm basically uses the same type of system with that of the NGMV but restricted to the structure of either a general ztransfer function or a PID. The cost function to be minimized also remains the same. The task of optimization deals with developing the optimal controller gains. As mentioned earlier, the NGMV control is a powerful technique for handling non-linear systems. It is indeed one of the simplest solutions offered for the control of non-linear systems. Therefore, providing an application of a novel NGMV controller with a structure that engineers in the industry are familiar to has been the driving force of this paper.

Linear Parameter Varying Systems (LPV Systems) are a special type of non-linear systems which can be represented as linear systems with parameters that change as the states change. The parameters can either be measured or estimated. A parameter is called exogenous if it is an external variable to the system and called endogenous if it is already a function of the state variables. The latter case is referred to as a quasiLPV (qLPV) system. They are often used to approximate non-linear systems [22, 23].

Robot arms owing to their wide range of applications from welding, assembly, painting, packaging in the industry to space and surgical operation systems have attracted the world wide attention of researchers and engineers. Due to coupled dynamics and a highly non-linear nature, the control of robotic arms might prove to be a tedious task. One way of handling the non-linearities is to express the non-linear system in what is called a state dependent form i.e. make it look like a linear model while maintaining its non-linear characteristics. For instance the qLPV representation of robotic manipulators has examples in the literature $[23-26]$ and has also been employed in this paper.

The paper is organized as follows. In Section II, the state-space representation of the system is provided. It is followed by the RS-NGMV control method in Section III. Then, Section IV covers the 2-link robot arm model along with the simulation studies comparing performances of PID and RS-NGMV controllers in position tracking objective and showing the time varying feedback gain deviations resulting from the optimization. Lastly, conclusions are given.

\section{STATE-SPACE SYSTEM DESCRIPTION}

\section{A. Augmented System Model}

In this section, an augmented $r \times m$ multivariable system is constructed using state dependent qLPV plant model and linear disturbance, weighted error models. Therefore, the state vector that corresponds to these models is represented as $x(t)=\left[\begin{array}{lll}x_{0}(t), & x_{d}(t), \quad x_{p}(t)\end{array}\right]^{T}$. Before going any further, 
it should be noted that the models that compose the augmented system will not be analyzed individually in this paper as they are already available [12]. The elements of the statedependent qLPV system are functions of states, inputs and parameters which are functions of the state variables. Thus the qLPV system appears in the form of $A_{0}\left(x_{0}(t), u_{0}(t-\right.$ $k), \rho(t))$. However, in order to avoid notational complexity it will be expressed by simply $A_{0 t}$. In the NGMV literature [4-13], the total plant model including linear and non-linear subsystems along with the delay operator is often denoted by $z^{-k} W_{0 k}\left(W_{1 k} u\right)(t)$ which contains the input sub-system,

$$
u_{0}(t)=\left(W_{1} u\right)(t)=z^{-k}\left(W_{1 k} u\right)(t) .
$$

For the analysis in this paper is only concerned with the control of a qLPV system, $W_{1 k}$ will be set to identity making $u_{0}(t)=u(t)$. Taking into consideration these facts, the augmented system model is demonstrated in the equation below,

$$
\begin{array}{r}
x(t+1)=\left[\begin{array}{ccc}
A_{0 t} & 0 & 0 \\
0 & A_{d t} & 0 \\
-B_{p t} C_{0 t} & -B_{p t} C_{0 t} & A_{p t}
\end{array}\right] x(t)+\left[\begin{array}{c}
B_{0 t} \\
0 \\
B_{p t} E_{0 t}
\end{array}\right] u_{0 k}(t-k) \\
+\left[\begin{array}{cc}
D_{0 t} & 0 \\
0 & D_{d t} \\
0 & 0
\end{array}\right]\left[\begin{array}{c}
\xi(t) \\
\omega(t)
\end{array}\right]+\left[\begin{array}{cc}
I & 0 \\
0 & 0 \\
0 & B_{p t}
\end{array}\right]\left[\begin{array}{c}
d_{0 d}(t) \\
(r(t)-d(t))
\end{array}\right] .
\end{array}
$$
by,

The weighted error $e_{p}(t)=P_{c}\left(z^{-1}\right) e(t)$ equation is given

$$
e_{p}(t)=d_{p}(t)+C_{p t} x(t)+E_{p t} u_{0}(t-k),
$$

where $P_{c}$ is an $r \times m$ size weighting operator that introduces the penalty on the errors.

\section{RS-NGMV CONTROL METHOD}

\section{A. Controller Structure}

The restricted structure controller is basically obtained from the multiplication of user pre-specified functions by some feedback gains. It can be formulated as follows:

$$
\begin{aligned}
u(t) & =\sum_{j=1}^{N_{e}} f_{j}\left(z^{-1}, k_{j}(t)\right) e_{0}(t)=\left(f_{1}\left(z^{-1}, k_{1}(t)\right) e_{0}(t)\right. \\
& \left.+f_{2}\left(z^{-1}, k_{2}(t)\right) e_{0}(t)+\cdots+f_{N_{e}}\left(z^{-1}, k_{N_{e}}(t)\right) e_{0}(t)\right),
\end{aligned}
$$

where $f_{j}\left(z^{-1}, k_{j}(t)\right)$ denotes the pre-specified functions and $k_{j}(t)$ represents the feedback gains. A typical example is the restricted structure PID control for a SISO system. In this case, the controller has $N_{e}=3$ function terms that can be chosen as $f_{1}\left(z^{-1}\right)=1, f_{2}\left(z^{-1}\right)=1 /\left(1-z^{-1}\right)$, $f_{3}\left(z^{-1}\right)=\left(1-z^{-1}\right) /\left(1-\alpha z^{-1}\right)$ which stand for the proportional, integral and the derivative terms respectively. The PID control input can then be computed by,

$$
u(t)=k_{1} e(t)+\frac{1}{1-z^{-1}} k_{2} e(t)+\frac{1-z^{-1}}{1-\alpha z^{-1}} k_{3} e(t) .
$$

For multivariable systems, it is much more rigorous to derive the restricted structure controller form. Consider an $r \times m$ multivariable system, under the assumption that $r \leq m$, the controller form in equation (4) expands as in,

$$
u(t)=\left[\begin{array}{c}
\sum_{j=1}^{N_{e}} \sum_{l=1}^{r}\left\{f_{11}^{j}\left(z^{-1}\right) k_{1 l}^{j} e_{0 l}(t)\right\} \\
\sum_{j=1}^{N_{e}} \sum_{l=1}^{r}\left\{f_{21}^{j}\left(z^{-1}\right) k_{2 l}^{j} e_{0 l}(t)\right\} \\
\vdots \\
\sum_{j=1}^{N_{e}} \sum_{l=1}^{r}\left\{f_{m 1}^{j}\left(z^{-1}\right) k_{m l}^{j} e_{0 l}(t)\right\}
\end{array}\right]_{m \times 1}
$$

The procedure to parameterize the controller $u(t)$ for multivariable systems can become easier by introducing following matrices of functions and gains. Re-arranging the elements from the rows of $u(t)$ starting from the first as in,

$\left[\begin{array}{c}f_{e}^{11} \\ f_{e}^{12} \\ \vdots \\ f_{e}^{1 r}\end{array}\right]=\left[\begin{array}{cccc}f_{11}^{1}\left(z^{-1}\right) e_{01}(t) & f_{11}^{2}\left(z^{-1}\right) e_{01}(t) & \ldots & f_{11}^{N_{e}}\left(z^{-1}\right) e_{01}(t) \\ f_{12}^{1}\left(z^{-1}\right) e_{02}(t) & f_{12}^{2}\left(z^{-1}\right) e_{02}(t) & \ldots & f_{12}^{N_{e}}\left(z^{-1}\right) e_{02}(t) \\ \vdots & \vdots & \ddots & \vdots \\ f_{1 r}^{1}\left(z^{-1}\right) e_{0 r}(t) & f_{1 r}^{2}\left(z^{-1}\right) e_{0 r}(t) & \ldots & f_{1 r}^{N_{e}}\left(z^{-1}\right) e_{0 r}(t)\end{array}\right]_{r \times N_{e}}$

and the second,

$\left[\begin{array}{c}f_{e}^{21} \\ f_{e}^{22} \\ \vdots \\ f_{e}^{2 r}\end{array}\right]=\left[\begin{array}{cccc}f_{21}^{1}\left(z^{-1}\right) e_{01}(t) & f_{21}^{2}\left(z^{-1}\right) e_{01}(t) & \ldots & f_{21}^{N_{e}}\left(z^{-1}\right) e_{01}(t) \\ f_{22}^{1}\left(z^{-1}\right) e_{02}(t) & f_{22}^{2}\left(z^{-1}\right) e_{02}(t) & \ldots & f_{22}^{N_{e}}\left(z^{-1}\right) e_{02}(t) \\ \vdots & \vdots & \ddots & \vdots \\ f_{2 r}^{1}\left(z^{-1}\right) e_{0 r}(t) & f_{2 r}^{2}\left(z^{-1}\right) e_{0 r}(t) & \ldots & f_{2 r}^{N_{e}}\left(z^{-1}\right) e_{0 r}(t)\end{array}\right]_{r \times N_{e}}$

and until the mth row,

$\left[\begin{array}{c}f_{e}^{m 1} \\ f_{e}^{m 2} \\ \vdots \\ f_{e}^{m r}\end{array}\right]=\left[\begin{array}{cccc}f_{m 1}^{1}\left(z^{-1}\right) e_{01}(t) & f_{m 1}^{2}\left(z^{-1}\right) e_{01}(t) & \ldots & f_{m 1}^{N_{e}}\left(z^{-1}\right) e_{01}(t) \\ f_{m 2}^{1}\left(z^{-1}\right) e_{02}(t) & f_{m 2}^{2}\left(z^{-1}\right) e_{02}(t) & \ldots & f_{m 2}^{N_{e}}\left(z^{-1}\right) e_{02}(t) \\ \vdots & \vdots & \ddots & \vdots \\ f_{m r}^{1}\left(z^{-1}\right) e_{0 r}(t) & f_{m r}^{2}\left(z^{-1}\right) e_{0 r}(t) & \ldots & f_{m r}^{N_{e}}\left(z^{-1}\right) e_{0 r}(t)\end{array}\right]_{r \times N_{e}}$

Then all they are all gathered in the matrix below,

$$
\left[\begin{array}{c}
e_{f 1}(t) \\
e_{f 2}(t) \\
\vdots \\
e_{f m}(t)
\end{array}\right]=\left[\begin{array}{cccc}
f_{e}^{11} & f_{e}^{12} & \ldots & f_{e}^{1 r} \\
f_{e}^{21} & f_{e}^{22} & \ldots & f_{e}^{2 r} \\
\vdots & \vdots & \ddots & \vdots \\
f_{e}^{m 1} & f_{e}^{m 2} & \ldots & f_{e}^{m r}
\end{array}\right]_{m \times r}
$$

and form the diagonal matrix $F_{e}(t)$,

$$
F_{e}(t)=\operatorname{diag}\left\{e_{f 1}(t), e_{f 2}(t), \cdots, e_{f m}(t)\right\} .
$$

Define a matrix $g_{c}(t)=\left[\begin{array}{llll}g_{c 1}(t) & g_{c 2}(t) & \ldots & g_{c m}(t)\end{array}\right]$ using elements of the gain $k_{c}(t)$ that consists of,

$$
\begin{gathered}
g_{c 1}(t)=\left[\begin{array}{c}
g_{c}^{11}(t) \\
g_{c}^{12}(t) \\
\vdots \\
g_{c}^{1 r}(t)
\end{array}\right]=\left[\begin{array}{cccc}
k_{11}^{1}(t) & k_{11}^{2}(t) & \ldots & k_{11}^{N_{e}}(t) \\
k_{12}^{1}(t) & k_{12}^{2}(t) & \ldots & k_{12}^{N_{e}}(t) \\
\vdots & \vdots & \ddots & \vdots \\
k_{1 r}^{1}(t) & k_{1 r}^{2}(t) & \ldots & k_{1 r}^{N_{e}}(t)
\end{array}\right]_{r \times N_{e}} \\
g_{c 2}(t)=\left[\begin{array}{c}
g_{c}^{21}(t) \\
g_{c}^{22}(t) \\
\vdots \\
g_{c}^{2 r}(t)
\end{array}\right]=\left[\begin{array}{cccc}
k_{21}^{1}(t) & k_{21}^{2}(t) & \ldots & k_{21}^{N_{e}}(t) \\
k_{22}^{1}(t) & k_{22}^{2}(t) & \ldots & k_{22}^{N_{e}}(t) \\
\vdots & \vdots & \ddots & \vdots \\
k_{2 r}^{1}(t) & k_{2 r}^{2}(t) & \ldots & k_{2 r}^{N_{e}}(t)
\end{array}\right]_{r \times N_{e}}
\end{gathered}
$$

up until,

$$
g_{c m}(t)=\left[\begin{array}{c}
g_{c}^{m 1}(t) \\
g_{c}^{m 2}(t) \\
\vdots \\
g_{c}^{m r}(t)
\end{array}\right]=\left[\begin{array}{cccc}
k_{m 1}^{1}(t) & k_{m 1}^{2}(t) & \ldots & k_{m 1}^{N_{e}}(t) \\
k_{m 2}^{1}(t) & k_{m 2}^{2}(t) & \ldots & k_{m 2}^{N_{e}}(t) \\
\vdots & \vdots & \ddots & \vdots \\
k_{m r}^{1}(t) & k_{m r}^{2}(t) & \ldots & k_{m r}^{N_{e}}(t)
\end{array}\right]_{r \times N_{e}}
$$


which will help construct the $\left(r \times m \times N_{e}\right) \times 1$ size vector of gains $k_{c}(t)$,

$$
k_{c}(t)=\left[\begin{array}{llll}
k_{c 1}(t) & k_{c 2}(t) & \cdots & k_{c m}(t)
\end{array}\right] .
$$

where $k_{c i}(t)=\left[\begin{array}{llll}g_{c}^{i 1}(t) & g_{c}^{i 2}(t) & \ldots & g_{c}^{i r}(t)\end{array}\right]$ with index $i=\{1,2, \ldots, m\}$. Finally, the control input for the multivariable system can be calculated by,

$$
u(t)=F_{e}(t) k_{c}(t)=\left[\begin{array}{c}
e_{f 1}(t) k_{c 1}(t) \\
e_{f 2}(t) k_{c 2}(t) \\
\vdots \\
e_{f m}(t) k_{c m}(t)
\end{array}\right]_{m \times 1}
$$

\section{B. Parallel Form of the Controller}

The restricted structure controller gain $k_{c}(t)$ can be divided into two components such as the constant component $\bar{k}_{c}$ and the time-varying deviation component $\widetilde{k}_{c}(t)$. The RS control input may then be re-arranged as in,

$$
\begin{aligned}
u(t) & =F_{e}(t) k_{c}(t)=F_{e}(t) \bar{k}_{c}+F_{e}(t) \widetilde{k}_{c}(t) \\
& =\sum_{j=1}^{N_{e}} f_{j}\left(z^{-1}, \bar{k}_{j}\right) e_{0}(t)+\sum_{j=1}^{N_{e}} f_{j}\left(z^{-1}, \widetilde{k}_{j}(t)\right) e_{0}(t) .
\end{aligned}
$$

There are two special cases concerning the gains. If $\bar{k}_{c}=0$, it is the absolute gain case and $k_{c}(t)=\widetilde{k}_{c}(t)$. If $\bar{k}_{c} \neq 0$, then $k_{c}(t)=\bar{k}_{c}+\widetilde{k}_{c}(t)$ and this is called the gain deviation case. Once again, the PID implementation could be considered as an example. The first case involves the minimization of the total PID controller gains. The second can resemble the case of having two parallel PID controllers one with constant gains, the other with time-varying gains which is especially practical if a PID controller is in hand already. The task is then to compute the deviations from the constant gains.

\section{Optimal RS-NGMV Control Law}

In this section the results from the analysis of the optimal RS-NGMV control law are demonstrated. Let us define the cost function to be minimized within the optimization task as,

$$
J=E\left\{\phi_{p}^{T}(t+k) \phi_{p}(t+k)\right\},
$$

which is the variance of the pseudo-output signal given by,

$$
\begin{aligned}
\phi_{p}(t+k)= & P_{p}(t) e_{p}(t+k)+F_{c 0} u_{0}(t)+F_{c 1} \widetilde{k}_{c}(t) \\
& +F_{c 2} \Delta \widetilde{k}_{c}(t)+F_{e}^{T}(t)\left(F_{c k} u\right)(t) .
\end{aligned}
$$

The signal $\phi_{p}(t+k)$ is composed of control and error weightings that are, $P_{p}(t)=F_{e}^{T}(t) E_{p t+k}^{T} \Lambda_{p}{ }^{2}, F_{c 0}=$ $F_{e}^{T}(t) \Lambda_{u}{ }^{2}, F_{c 1}=\Lambda_{k}{ }^{2}, F_{c 2}=\Lambda_{d}{ }^{2}, F_{c k}$. The dynamic weighting on tracking error is weighted by the constant matrix $\Lambda_{p}{ }^{2} \in \mathcal{R}^{r \times m}$, the constant weightings on the control inputs is defined as $\Lambda_{u}{ }^{2} \in \mathcal{R}^{r \times m}$, the constant weightings on the deviations in controller gains is defined as $\Lambda_{k}{ }^{2} \in$ $\mathcal{R}^{(r \times m) \times N_{e}}$ diagonal matrix and the cost weighting on the increments on the deviations in gains is denoted $\Lambda_{d}^{2} \in$ $\mathcal{R}^{(r \times m) \times N_{e}}$ diagonal matrix. The control weighting operator $F_{c k}$ is supposed to be full rank and invertible and can be non-linear. It takes the form $\left(F_{c} u\right)(t)=z^{-k}\left(F_{c k} u\right)(t)$.
Under the assumption that the plant is of the qLPV and $W_{1 k}=I$, the controller gain that will minimize the cost function $J$ will be calculated as,

$$
\left.k_{c}(t)=-X_{0}(t)^{-1}\left(P p(t) d_{p d}^{0}(t+k)\right)+\psi_{k}(t)\right),
$$

where terms $\psi_{k}(t)=-\Lambda_{k}^{2} \bar{k}_{c}-\Lambda_{d}^{2} k_{c}(t-1), d_{p d}^{0}(t+k)=$ $d_{p d}(t+k)+C_{p t+k} \hat{x}(t+k \mid t)$ and $X_{0}(t)$ is a time-varying real symmetric matrix defined by,

$$
\begin{aligned}
X_{0}(t)= & F_{e}^{T}(t)\left(F_{c k}+\left(E_{p t+k}^{T} \Lambda_{p} E_{p t+k}+\Lambda_{u}{ }^{2} W_{1 k}\right) F_{e}(t)\right. \\
& +\Lambda_{k}{ }^{2}+\Lambda_{d}{ }^{2} .
\end{aligned}
$$

Then the optimal RS-NGMV controller $u(t)=F_{e}(t) k_{c}(t)$ can be implemented as shown in Figure 1,

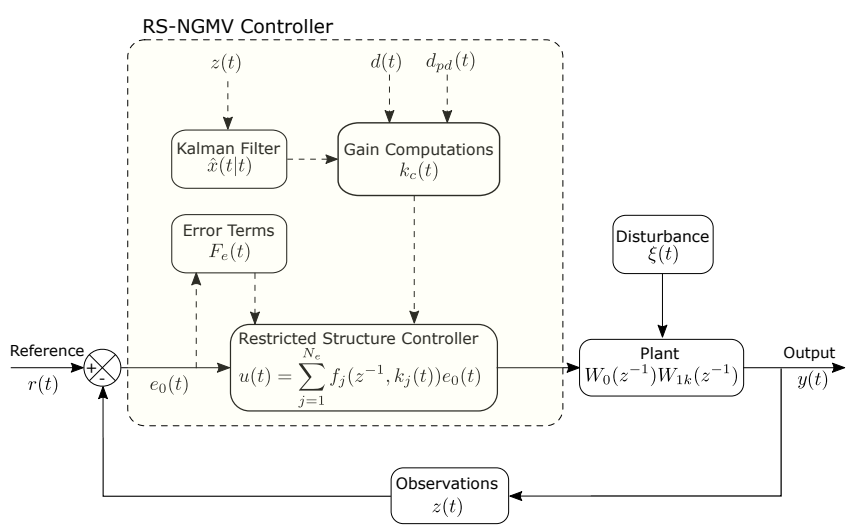

Figure 1: RS-NGMV control block diagram.

representing the algorithm. The full derivation and solution of the controller gains $k_{c}(t)$ will be available in [17], submitted for publication.

\section{Prediction Model}

For the online gain computation of the RS-NGMV controller, a Kalman filter is used to estimate states $\hat{x}(t \mid j)$. The notation $\hat{x}(t \mid j)$ means that the estimate of $\hat{x}(t)$ based on all information up to and including the time $j$. The Kalman filter considers delays if they are present in the system thus a prediction model is needed. The $k$-steps ahead predictor may be implemented as in the equation,

$$
\begin{aligned}
\hat{x}(t+k \mid t) & =A_{t}^{k} \hat{x}(t \mid t)+\sum_{j=1}^{k} A_{t+j}^{k-j} B_{t+j-1} u_{0}(t+j-1-k) \\
& +d_{d d}(t+k-1),
\end{aligned}
$$

which is obtained by using (2) iteratively. The full derivation process of the predictor is available in [12]. The $k$-steps ahead prediction errors can be calculated in a similar fashion,

$$
\hat{e}(t+k \mid t)=d_{p}(t+k)+C_{p t+k} \hat{x}(t+k \mid t)+E_{p t+k} u_{0}(t) .
$$




\section{ROBOT ARM EXAMPLE}

The robotic manipulator (see Figure 2) used for our example is composed of two rigid links and two revolute joints. Euler-Lagrange approach has been taken for modelling of it's dynamics as given more in detail in $[18-21]$. With the help of the Lagrangian equations robot's dynamics can be expressed by,

$$
M(q) \ddot{q}+C(q, \dot{q}) \dot{q}+F(\dot{q})+G(q)=\tau,
$$

where $M(q)$ represents the inertia matrix and $C(q, \dot{q})$, $F(\dot{q}), G(q)$ represent the Coriolis matrix, friction and gravity vectors respectively. Torque is expressed by the vector $\tau$ while $q$ denotes the joint angle vector.

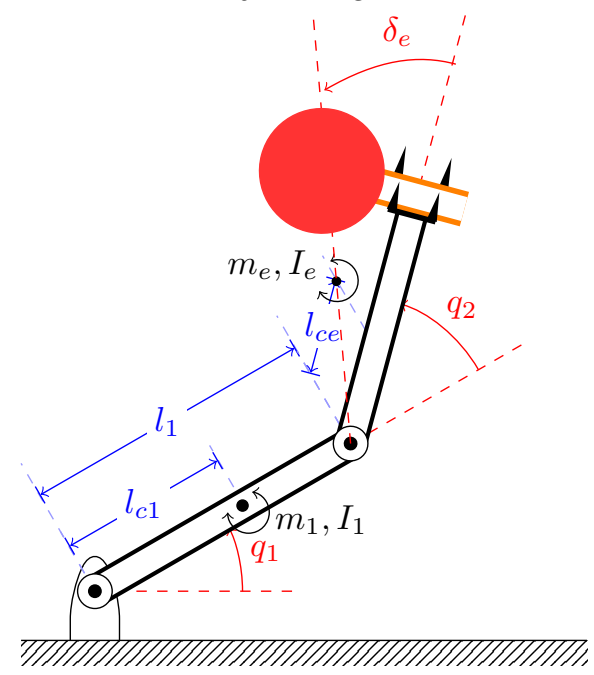

Figure 2: Two-link robot arm.

\section{A. System Model of the 2-link Robot Arm}

In this study the forces of friction are neglected and it is assumed that the robot is operating horizontally, i.e. moving on 2-dimensional Euclidean plane which means that the affect of gravitational forces can be further ignored. In this regard, expanding the dynamics in the matrix form for the 2-link robot manipulator will yield to the equation,

$$
\left[\begin{array}{ll}
M_{11} & M_{12} \\
M_{21} & M_{22}
\end{array}\right]\left[\begin{array}{l}
\ddot{q}_{1} \\
\ddot{q}_{2}
\end{array}\right]+\left[\begin{array}{cc}
h \dot{q}_{2} & h \dot{q}_{1}+h \dot{q}_{2} \\
h \dot{q}_{1} & 0
\end{array}\right]\left[\begin{array}{l}
\dot{q}_{1} \\
\dot{q}_{2}
\end{array}\right]=\left[\begin{array}{l}
\tau_{1} \\
\tau_{2}
\end{array}\right] \text {. }
$$

The 2-link robot has strong non-linear characteristics due to the nature of equation (18). The non-linearity becomes more apparent when the elements of the inertia matrix $M$ are analyzed,

$$
\begin{aligned}
& M_{11}=\rho_{1}+2 \rho_{3} \cos _{2}+2 \rho_{4} \sin _{2}, \\
& M_{12}=M_{21}=\rho_{2}+\rho_{3} \cos q_{2}+\rho_{4} \sin _{2}, \\
& M_{22}=\rho_{2},
\end{aligned}
$$

with parameters,

$$
\begin{aligned}
& \rho_{1}=I_{1}+m_{1} l_{c 1}^{2}+I_{e}+m_{e} l_{c e}^{2}+m_{e} l_{1}^{2}, \\
& \rho_{2}=I_{e}+m_{e} l_{c e}^{2}, \\
& \rho_{3}=m_{e} l_{1} l_{c e} \cos \delta_{e}, \\
& \rho_{4}=m_{e} l_{1} l_{c e} \sin \delta_{e},
\end{aligned}
$$

and finally $h=\rho_{3} \operatorname{sinq}_{2}-\rho_{4} \cos q_{2}$ of the Coriolis matrix $C$. Since, the inertia matrix $M$ is invertible, after some rearranging of the equations it is possible to represent the system in the state-space form by (note that $q=\left[q_{1}, q_{2}\right]^{T}$ and $\tau=\left[\tau_{1}, \tau_{2}\right]^{T}$ ),

$$
\begin{gathered}
\dot{x}=\left[\begin{array}{c}
\dot{q} \\
\ddot{q}
\end{array}\right]=\left[\begin{array}{cc}
0 & I \\
0 & M^{-1}(q) C(\dot{q})
\end{array}\right]\left[\begin{array}{c}
q \\
\dot{q}
\end{array}\right]+\left[\begin{array}{c}
0 \\
M^{-1}(q)
\end{array}\right] \tau . \\
y=q=\left[\begin{array}{ll}
I & 0
\end{array}\right]\left[\begin{array}{c}
q \\
\dot{q}
\end{array}\right] .
\end{gathered}
$$

\section{B. Simulation Results}

Robot parameters used within this simulation study have been adopted from example 9.1 in [20]. They are given by, $m_{1}=1, I_{1}=0.12, l_{1}=1, l_{c 1}=0.5, m_{e}=2, I_{e}=$ $0.25, l_{c e}=0.6, \delta_{e}=30^{\circ}$. The control objective is to have the robot follow a desired position trajectory $q_{d}=\left[q_{1 d}, q_{2 d}\right]$ which is specified in detail as,

$$
q_{d}= \begin{cases}{\left[60^{\circ}, 90^{\circ}\right],} & \text { for } t \leq 50 \\ {\left[45^{\circ}, 45^{\circ}\right],} & \text { for } 50<t \leq 100 \\ {\left[60^{\circ}, 90^{\circ}\right],} & \text { for } t>100\end{cases}
$$

by sending torque signals to the joints and saturation limits $\pm 2 \mathrm{Nm}$ is applied to torque inputs. The inputs are subject to the torque disturbance of $T_{d}=0.4 \mathrm{Nm}$. The sampling time for the simulation is $T_{s}=0.005 \mathrm{~s}$.

In the design of the RS-NGMV controller, the second case of the parallel form in equation (10) is used which implies that a PID controller already exists for the robot whose parameters are chosen as,

$$
\bar{k}_{c}=\left[\begin{array}{l}
k_{p} \\
k_{I} \\
k_{D}
\end{array}\right]=\left[\begin{array}{c}
3 \\
0.5 \\
7
\end{array}\right] .
$$

In the implementation of the controller time-varying gain deviations $\widetilde{k}_{c}(t)$ will be calculated and added to the constant gains.

The error and control cost weightings are specified,

$$
\begin{aligned}
& P_{c}\left(z^{-1}\right)=\left[\begin{array}{cc}
87.5 \frac{1-0.97 z^{-1}}{1-z^{-1}} & 0 \\
0 & 87.5 \frac{1-0.97 z^{-1}}{1-z^{-1}}
\end{array}\right], \\
& F_{c k}\left(z^{-1}\right)=\left[\begin{array}{cc}
0.016 \frac{1-0.9 z^{-1}}{1-0.4 z^{-1}} & 0 \\
0 & 0.16 \frac{1-0.9 z^{-1}}{1-0.4 z^{-1}}
\end{array}\right] \text {, } \\
& \Lambda_{k}^{2}=10^{-6} \times \operatorname{diag}\{[[3,0.5,7], \cdots, \quad[3,0.5,7]]\}, \\
& \Lambda_{d}^{2}=40 \times \operatorname{diag}\{[[1,1,1], \cdots, \quad[1,1,1]]\}, \\
& \Lambda_{p}^{2}=\left[\begin{array}{cc}
10^{-4} & 0 \\
0 & 10^{-4}
\end{array}\right], \Lambda_{u}^{2}=\left[\begin{array}{cc}
0.05 & 0 \\
0 & 0.05
\end{array}\right] \text {. }
\end{aligned}
$$


The simulation results are given in Figures 3 and 4. First, the position tracking performances of RS-NGMV and PID are compared in Figure 3.
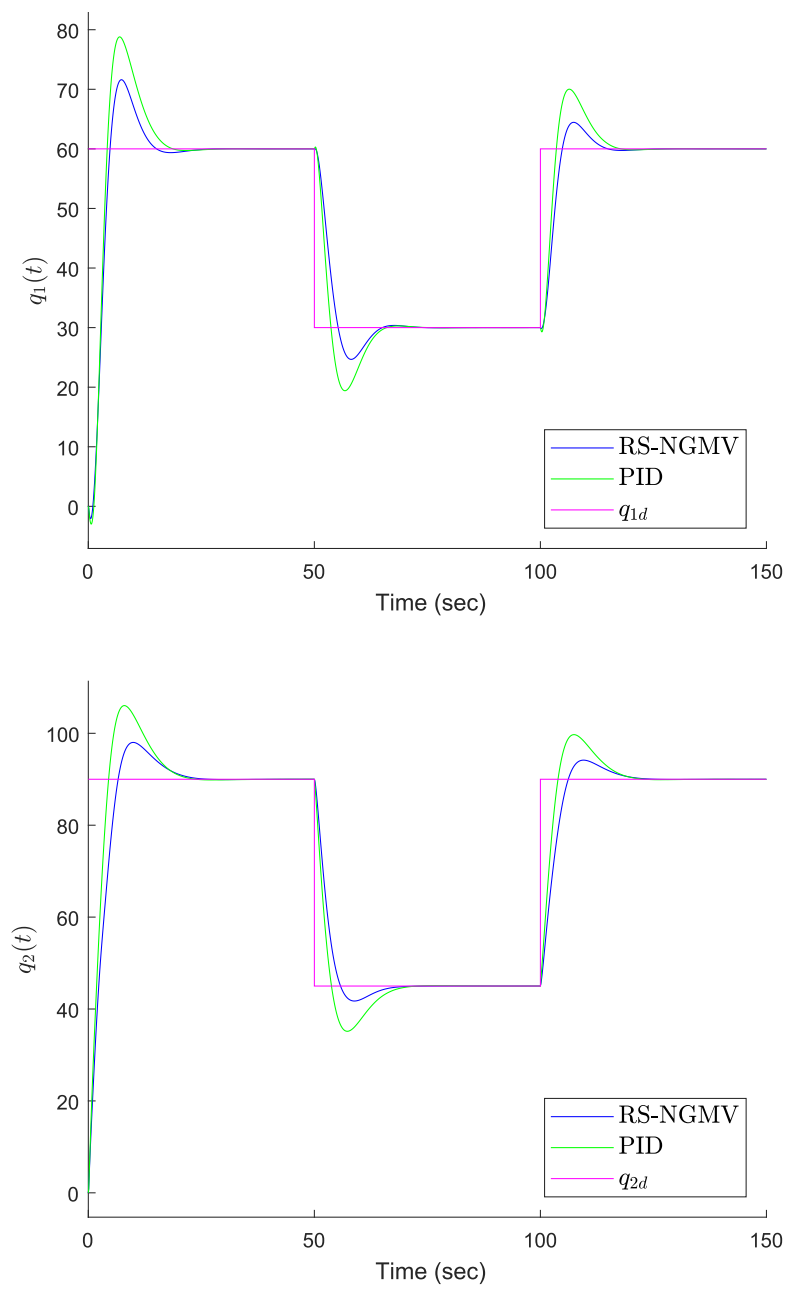

Figure 3: Results for the position tracking.

It demonstrates the control efforts in following the reference angle trajectory for links 1 and 2 respectively. As can be observed from the figure, the results show that both RSNGMV and PID controllers achieve the control objective of the desired joint angle tracking for the 2-link robot arm. RS-NGMV owing to the advantages inherited from the NGMV as an optimal control solution, shows slightly better performance compared to the PID with less overshoot. The RS-NGMV controller also adapts easier to the setpoint changes thanks to the optimized feedback gains. It is demonstrated that tuning procedure is actually familiar for designers with background in PID control.

In Figure 4 , the time varying gain deviations $\widetilde{k}_{c}(t)$ resulting from the optimization process are illustrated. It is shown that they are constantly updated for the feedback gains of the RS-NGMV controller. For example, much calculation effort is spent at and around time $t=0$ as the link angles $q_{1}(t)$ and $q_{2}(t)$ try to catch their reference angles. The deviations $\widetilde{k}_{c}(t)$ tend to slow down as the system output reaches the steady state until the next reference angle signals are generated. Then the same pattern is repeated.
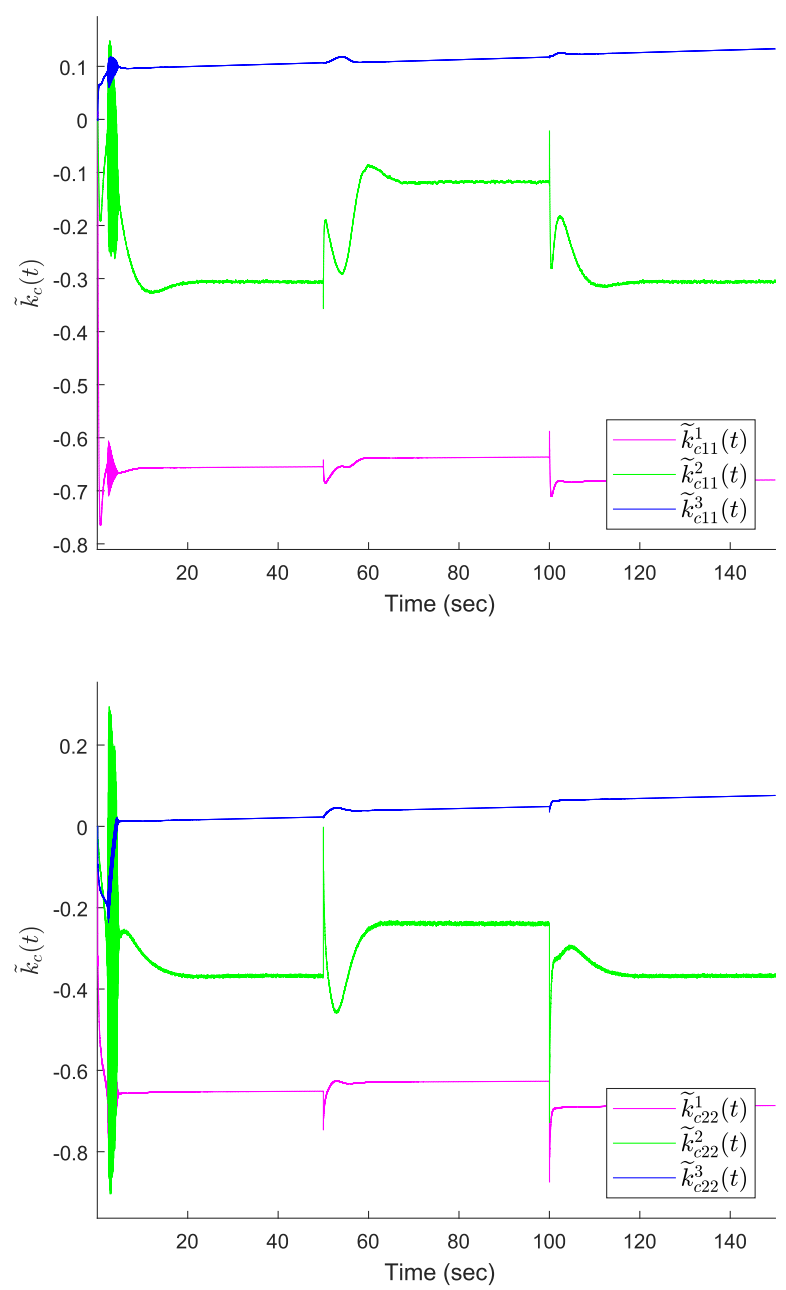

Figure 4: Time varying gain deviations.

\section{CONCLUSIONS}

In this work, a multivariable RS-NGMV controller has been designed for the qLPV model of a 2-link robotic arm. The controller has been set to have the structure of a PID and employs optimized time varying feedback gains that are constantly updated. The gains help the RS-NGMV control algorithm adapt to the changes such as reference trajectories much easily. Performance comparison of the controller to that of a classical PID controller has been made and the results show that it works better.

\section{ACKNOWLEDGMENT}

The author C. Cebeci would like to express his gratitude to Turkish Ministry of National Education for the sponsorship of his studies. 


\section{REFERENCES}

[1] K. J. Åström, Introdution to Stochastic Control Theory, Dover Publications, 2006.

[2] D.W. Clarke and R. Hastings-James, "Design of digital controllers for randomly disturbed systems", Proc. IEEE, Vol. 118, pp. 1503-1506, 1971.

[3] P. Wellstead and M. Zarrop, Self-Tuning Systems: Control and Signal Processing, Wiley, 1991.

[4] M. J. Grimble, "GMV control of non-linear multivariable systems, UKACC Control Conference", 2004.

[5] M. J. Grimble, "Non-linear generalized minimum variance feedback, feedforward and tracking control", Automatica, Vol.41, pp. 957-969, 2005.

[6] M. J. Grimble, "Design of generalized minimum variance controllers for nonlinear systems", International Journal of Control, Automation and Systems, Vol.4, pp. 281-292, 2006.

[7] M. J. Grimble and Y. Pang, "NGMV control of state dependent multivariable systems", IEEE CDC Conference, pp. 1628-1633, 2007.

[8] M. J. Grimble and P. Majecki, "Nonlinear predictive GMV control", American Control Conference, pp. 1190-1195, 2008.

[9] Y. Pang and M.J. Grimble, "State dependent NGMV control of delayed piecewise affine systems", IEEE CDC Conference, pp. 7192-7197, 2009.

[10] M. J. Grimble and P. Majecki, "Polynomial approach to non-linear predictive generalised minimum variance control", IET Control Theory and Applications, pp. 411-424, 2009.

[11] M. J. Grimble and P. Majecki, "State-space approach to nonlinear predictive generalised minimum variance control", International Journal of Control, Vol. 83, pp. 1529-1547, 2010.

[12] M. J. Grimble and P. Majecki, "Non-linear predictive generalised minimum variance state-dependent control", IET Control Theory \& Applications, pp. 1-13, 2015.

[13] P. Savvidis, Nonlinear control : an LPV nonlinear predictive generalised minimum variance perspective, $\mathrm{PhD}$ Thesis, Dept. of Electronic \& Electrical Eng., University of Strathclyde, 2017.
[14] M. J. Grimble, "Restricted structure predictive optimal control", Optimal Control Applications and Methods, Vol. 25, no. 3, pp. 107-145, 2004.

[15] M. H. Moradi, M. R. Katebi and M. A. Johnson, "Predictive PID control: A new algorithm", IECON'O1: The 27th Annual Conference of the IEEE Industrial Electronics Society, Vol. 1, pp. 764-769, 2001.

[16] M. A. Johnson, M. H. Moradi, "PID control: New Identification and Design Methods", 1st ed., Springer-Verlag London, 2005.

[17] M. J. Grimble, "Reduced order nonlinear generalized minimum variance control for qLPV systems", Research Report: ICC-244, Industrial Systems and Control Limited, Glasgow, United Kingdom, Submitted for publication, 2018.

[18] M. W. Spong, S. Hutchinson and M. Vidyasagar, Robot Modeling and Control, 1st ed., New York: John Wiley \& Sons, 2005.

[19] F. L. Lewis, D. M. Dawson and C. T. Abdallah, Robot Manipulator Control: Theory and Practice, 2nd ed., New York: Marcel Dekker, 2004.

[20] J. J. E. Slotine and W. Li, Applied Nonlinear Control, Englewood Cliffs, New Jersey: Prentice Hall, 1991.

[21] K. M. Lynch and F. C. Park, Modern Robotics: Mechanics, Planning, and Control, Cambridge U. Press, 2017.

[22] C. Briat, Linear Parameter Varying and Time-delay Systems: Analysis, Observation, Filtering \& Control, Advances in Delay and Dynamics, Vol.3, Springer-Verlag Berlin Heidelberg, 2015.

[23] A. A. G. Siqueira, M. H. Terra and M. Bergerman, Robust Control of Robots: Fault Tolerant Approaches, Springer-Verlag London, 2011.

[24] H. Abbas, S. M. Hashemi and H. Werner, "Decentralized LPV gainscheduled PD control of a robotic manipulator", Proc. ASME Dynamic Systems and Control Conference, 2009.

[25] S. M. Hashemi, U. Gürcüoğlu, H. Werner, "Interaction control of an industrial manipulator using LPV techniques", Mechatronics, Vol. 23, pp. 689699, 2013.

[26] M. Löhning, "LPV and LFR modelling of elastic robots for controller synthesis", 8th IEEE International Conference on Control and Automation, pp. 522-527, 2010 